\title{
OPEN Maintenance of prior behaviour can enhance cultural selection
}

\author{
Bradley Walker ${ }^{1}$, José Segovia Martín ${ }^{2}$, Monica Tamariz ${ }^{3} \&$ Nicolas Fay ${ }^{1 凶}$
}

Many cultural phenomena evolve through a Darwinian process whereby adaptive variants are selected and spread at the expense of competing variants. While cultural evolutionary theory emphasises the importance of social learning to this process, experimental studies indicate that people's dominant response is to maintain their prior behaviour. In addition, while payoff-biased learning is crucial to Darwinian cultural evolution, learner behaviour is not always guided by variant payoffs. Here, we use agent-based modelling to investigate the role of maintenance in Darwinian cultural evolution. We vary the degree to which learner behaviour is payoff-biased (i.e., based on critical evaluation of variant payoffs), and compare three uncritical (non-payoff-biased) strategies that are used alongside payoffbiased learning: copying others, innovating new variants, and maintaining prior variants. In line with previous research, we show that some level of payoff-biased learning is crucial for populations to converge on adaptive cultural variants. Importantly, when combined with payoff-biased learning, uncritical maintenance leads to stronger population-level adaptation than uncritical copying or innovation, highlighting the importance of maintenance to cultural selection. This advantage of maintenance as a default learning strategy may help explain why it is a common human behaviour.

Many human traits, including language, religion and cooking, are culturally inherited ${ }^{1-3}$. We acquire these traits through social learning, and they evolve over time ${ }^{4-6}$. In many cases culture evolves through a Darwinian process where more adaptive variants selectively spread through the population at the expense of less adaptive variants ${ }^{7}$. Historical examples include the evolution of stone tools ${ }^{8}$, urban legends ${ }^{9}$, violin sound holes ${ }^{10}$, and aeroplane landing gear ${ }^{11}$. Experimental simulations have been used to demonstrate Darwinian cultural evolution under controlled laboratory conditions. Here, small groups of human participants produce material or symbolic artefacts such as stone tools ${ }^{12}$, arrowheads $s^{13-15}$, paper planes ${ }^{16,17}$ and communication systems ${ }^{18-20}$ that adaptively improve over time.

When learning in these studies, and in general, people can switch between three basic strategies: they can copy a variant from someone else (copying), innovate a new variant (innovation), or reuse a prior variant (maintenance). Whereas cultural evolutionary theory emphasises the importance of social learning (i.e., copying $)^{1,4,5,21}$, a striking finding from experimental studies is that people's dominant response is to maintain their prior behaviour ${ }^{22-25}$ (also known as egocentric discounting ${ }^{22,26,27}$ and behavioural conservatism ${ }^{28-30}$ ). Researchers have struggled to explain this ${ }^{27}$. Given the dominance of maintenance in human behaviour, it is important to examine the role it might play in Darwinian cultural evolution. If maintenance confers a benefit over other learning strategies, this may help to explain why it is common.

One reason people might maintain a prior variant is because it has a higher payoff than other variants. This can be considered a form of payoff-biased learning, a term typically applied in the context of social learning ${ }^{31-33}$ (i.e., copying based on variant payoffs). Asocial learning can also be considered payoff-biased, in the sense that it involves switching between innovation and maintenance based on variant payoffs (i.e., the "error" in "trial and error"). Mathematical and computational modelling have shown that payoff-biased learning is necessary for the selective spread of adaptive variants in a population ${ }^{34-38}$. These models show that payoff-biased learning increases the adaptiveness of culture through adaptive filtering ${ }^{35}$ : learners critically evaluate the variants they encounter, and are guided by a payoff bias to adopt adaptive variants and discard maladaptive variants. This filters maladaptive variants out of the population, increasing the probability that the variants that spread through social learning will be adaptive.

While payoff-biased learning is necessary for adaptive culture, variant adoption is not always guided by payoffs: a significant proportion of people maintain their own variants despite evaluating other variants as having higher payoffs ${ }^{26,39}$. Furthermore, there is evidence that people do not always evaluate variant payoffs, declining

\footnotetext{
${ }^{1}$ School of Psychological Science, University of Western Australia, 35 Stirling Highway, Crawley, WA 6009, Australia. ${ }^{2}$ Centre National de la Recherche Scientifique (CNRS), Institut des Systèmes Complexes Paris Île-de-France (ISC-PIF), Paris, France. ${ }^{3}$ Department of Psychology, Heriot-Watt University, Edinburgh, UK. ${ }^{\square}$ email: nicolas.fay@gmail.com
} 


\begin{tabular}{|l|l|l|}
\hline Parameter/variable & Symbol & Value(s) \\
\hline Payoff bias & $b$ & $0,0.2,0.4,0.6,0.8,1$ \\
\hline Learner type & $t$ & Copier, innovator, maintainer \\
\hline Error rate & $e$ & 0.02 \\
\hline Variant pool size (number of possible variants) & & 100 \\
\hline Population size & & $10,20,100,200$ \\
\hline Population structure & & Random, neighbourhoods of approximately $6,16,25,33$ agents \\
\hline Time steps & & 200 \\
\hline
\end{tabular}

Table 1. Model parameters and variables.

to even access the variants produced by others ${ }^{40}$. One reason for this might be that payoff evaluation is costly: there may be health risks involved (e.g., assessing an unfamiliar power tool), and learners do not always have the resources available, in terms of time, energy, motivation or expertise. This is recognised in cultural evolutionary theor $y^{4,41,42}$, which includes low-cost strategies for indirectly estimating payoffs based on features such as model prestige $^{43,44}$ or majority size ${ }^{45,46}$. However, these low-cost strategies are not used reliably ${ }^{32,47,48}$, and researchers have overlooked uncritical strategies that incur no evaluation cost at all. Uncritical strategies alone cannot lead to adaptive culture, but if learning is sometimes payoff-biased overall costs may be reduced by reverting to uncritical "default" strategies (i.e., arbitrarily copying, innovating or maintaining without estimating payoffs; see Tamari $\mathrm{z}^{49}$ ). There is some evidence that uncritical maintenance plays a role in cultural selection during the development of novel communication systems ${ }^{50}$, though it remains unclear how Darwinian cultural evolution would be impacted by different uncritical strategies.

In the present paper we use agent-based modelling to test how Darwinian cultural evolution may be impacted by maintenance, in comparison to copying and innovation, when varying the degree to which learners are critical (i.e., the degree to which they engage in payoff-biased learning). To test the robustness of our results, we examine unstructured populations with a range of sizes (10,20,100 and 200 agents), as well as structured 200-agent populations with a range of neighbourhood sizes (neighbourhoods of approximately 6, 16, 25 and 33 agents). In line with previous research, we show that some level of payoff-biased learning is crucial for populations to converge on adaptive variants. Importantly, when learning is not always critical, a default strategy to uncritically maintain prior behaviour improves the cultural selection process relative to uncritical copying and innovation.

\section{Method}

Purpose. The purpose of the model is to understand how Darwinian cultural evolution is affected by the degree to which learners are critical, adopting variants in a payoff-biased manner, and by whether they copy, innovate or maintain when uncritical. We compare variant diversity and adaptation across homogeneous populations of agents who copied when uncritical, innovated when uncritical or maintained when uncritical.

The model. The model consists of populations of agents, characterised by the variables shown in Table 1 . On each of a series of 200 time steps, the task for each agent in a population was to choose a cultural variant to carry into the next time step (agents possessed one variant at a time, with no memory of earlier variants). There were three sources of variants: agents could (1) copy the variant possessed by another agent in their population (i.e., a model), (2) innovate a new variant, or (3) maintain their variant from the previous time step. Agents chose between copying, innovation and maintenance based on variant payoffs ("Variants and variant payoffs" section) when they were critical (i.e., guided by payoff bias, "Payoff bias" section). When uncritical, the choice was determined by an agent's learner type ("Learner type" section). There was also a possibility of producing the wrong variant through error (error rate, "Error rate" section). To test the robustness of the results, we simulated populations in a variety of sizes and with different neighbourhood structures ("Population size and structure" section).

Process overview. At the start of each run, a population was generated with a variant pool (100 variants with associated payoffs, representing all possible variants) and a list of agents. All the agents in the population were initialised with a random variant sampled with replacement from the population's variant pool. All agents in the population were given the same payoff bias, learner type and error rate parameter values.

At each of 200 time steps, each agent in the population was given the opportunity to innovate a new variant, copy the variant of another agent in the population (a model), or maintain their prior variant (or make an error and adopt a random variant). This process was sequential (i.e., a later agent could copy from an earlier agent whose variant had already changed during that time step), and the same model could be selected by multiple agents. The structure of agents' choices, as determined by the model parameters, is shown in Fig. 1. Payoff bias $b$, learner type $t$, and error rate $e$ were set when running the simulations, while the payoff of the new, innovated variant $p_{n}$, the payoff of the model's variant $p_{m}$, and the payoff of the agent's own variant $p_{s}$ depended on the population's payoff distribution. The equations that determined agent behaviour from these parameters are shown in Supplementary Information S1.

Variants and variant payoffs. Each population was initialised with a variant pool containing 100 variants, representing all possible variants. Agents' variants were sampled from this pool. Following Rendell et al. ${ }^{51}$, variant 


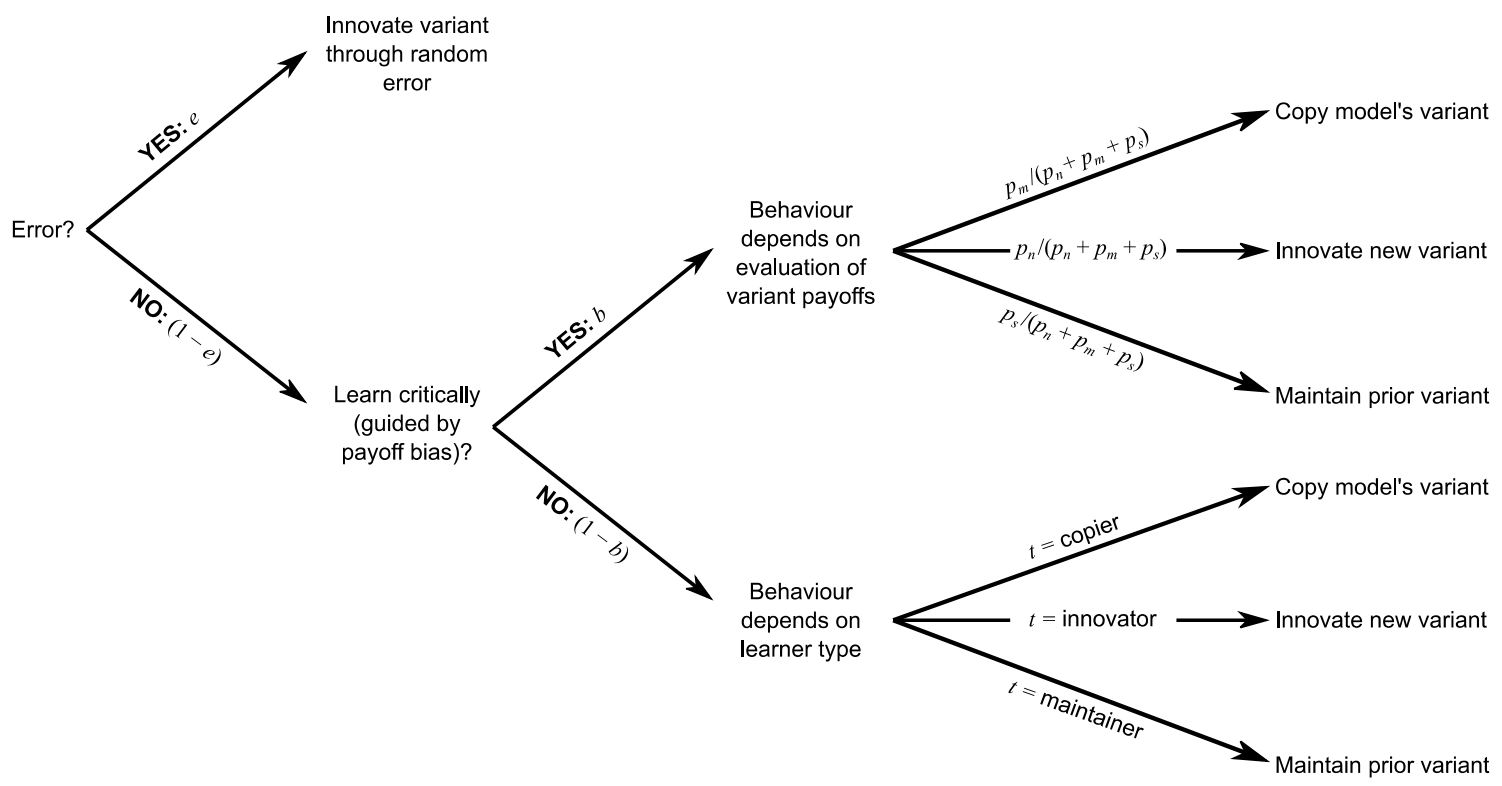

Figure 1. Diagram showing how the various parameters drove agents' variant choices.

payoffs were determined by an exponential distribution. This type of distribution is considered realistic, as it gives a small number of variants with very high payoffs, and a majority of variants with (very) low payoffs. Each variant in the pool was represented arbitrarily by an integer between 0 and 99 , and was associated with a payoff determined by sampling a random value from an exponential distribution $(\lambda=1$; as per Rendell et al., values were then squared, doubled and rounded, and 1 was added to avoid division by zero errors; this gave a minimum payoff of 1 and a maximum payoff typically around 50). New variant payoffs were generated for each run.

Payoff bias. The payoff bias parameter $(b)$ varied over 6 levels, in increments of 0.2 from 0 (fully uncritical) to 1 (fully critical). This parameter represented the probability with which an agent would behave critically, choosing between variants based on their evaluated payoffs (e.g., $20 \%$ of the time when payoff bias was 0.2 ). The model was agnostic with respect to whether agents evaluated payoffs directly (e.g., by experimentation) or indirectly (e.g., by estimating others' payoffs or through forward modelling). When agents were guided by payoff bias, the probability of choosing each variant was proportional to the variant's payoff. For instance, given variants with payoffs of 10,6 and 4 , the variant with payoff 10 would be chosen with a probability of $0.5(\text { i.e., } 10 /(10+6+4))^{52}$. When agents were not guided by payoff bias (i.e., were uncritical), their behaviour was determined by their learner type.

Learner type. The learner type parameter $(t)$ defined agents' uncritical default behaviour, so that a learner could either be a copier, an innovator, or a maintainer (though note that learner type did not vary within populations). When payoff bias was 0 , agent behaviour was never guided by variant payoffs, and was fully dictated by learner type: a copier would always copy, an innovator would always innovate, and a maintainer would always maintain their prior variant, regardless of variant payoffs (this simulated cultural drift under the different learner types). When payoff bias was 1, agent behaviour was always based on variant payoffs and learner type never came into play, so copiers, innovators and maintainers behaved identically under this parameter setting. At intermediate payoff bias levels, agents would be guided by payoff bias some of the time and at other times revert to the default behaviour determined by their learner type.

Error rate. The error rate parameter (e) represented all kinds of errors that could lead a learner to produce the wrong variant (e.g., copying error, production error), and was set at 0.02 , as this was the error rate in past cultural transmission experiments and used in previous models ${ }^{50,53,54}$. When agents produced the wrong variant through error, they adopted a variant randomly sampled from the population's variant pool (including the variant they were trying to produce).

Population size and structure. We first simulated populations of various sizes, with 10, 20, 100 and 200 agents each. These populations were unstructured, with agents' models (for the purposes of copying) selected at random from the whole population. As large unstructured populations are unrealistic, and to test the robustness of our results, we next imposed structure on populations of 200 agents by selecting models from the "neighbourhood" around each agent using Gaussian distributions, following Barr ${ }^{55}$. The agents were treated as being evenly distributed on a line, and a model was selected by sampling from a Gaussian distribution centred on an agent (and not allowing an agent to be their own model), so that increased distance from an agent reduced the probability of being selected as their model (see Fig. 2). The line was assumed to be circular (so the first and last agents 


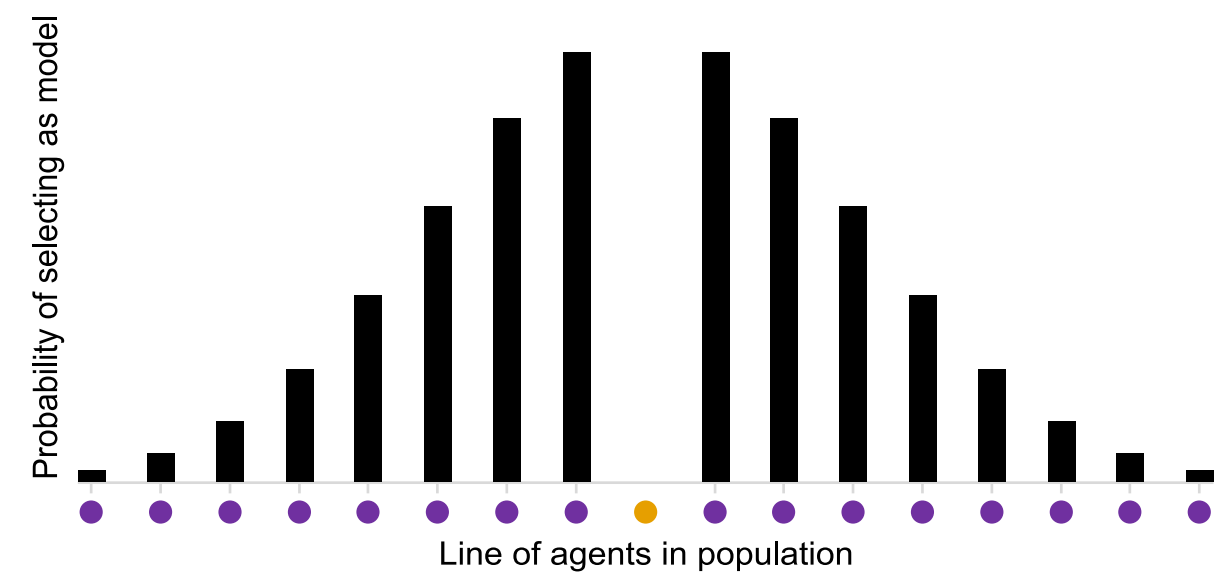

Figure 2. Diagram showing how neighbourhood structure was imposed by selecting each agent's (in orange) models from the neighbouring agents (in purple) using Gaussian distributions.

in the population were neighbours). We used Gaussian distributions with standard deviations of 1, 3, 5 and 7 to create neighbourhood sizes of approximately 6,16,25 and 33 agents respectively, representing the number of models an agent had access to over the simulated time period.

Quantifying variant diversity and adaptation. To measure the effect of payoff-biased learning and the default uncritical behaviours on Darwinian cultural evolution, population-level variant diversity and average payoff were measured at the end of each time step. For variant diversity, we used the Gini-Simpson index of diversity $^{56}$, an ecological measure previously applied to culture ${ }^{57-59}$. With this measure, scores close to 1 indicate high diversity (agents using different variants) and a score of 0 indicates no diversity (all agents sharing the same variant). To calculate a population's average payoff, we simply averaged the payoffs of variants possessed by the agents in the population. When the average payoff increased over time, we considered a population to be demonstrating adaptation.

\section{Results}

The first set of simulations examined the influence of the different parameter combinations on cultural variant diversity and adaptation in populations of different sizes $(10,20,100$ and 200 agents). The populations were unstructured: every agent in the population had an equal probability of being selected as a model for the purposes of copying. We ran 10,000 simulations for each parameter combination.

Variant diversity decreased over time as agents converged on a reduced set of cultural variants. Convergence was faster and stronger as payoff bias increased. Smaller populations showed the strongest convergence (though not necessarily on the most adaptive variants). Population-level convergence was moderated by learner type: copiers converged most strongly, with maintainers retaining more diversity, and innovators continually generating more diversity and converging the least (see Fig. 3a).

Variant adaptation required payoff-biased learning. As payoff bias increased, populations increasingly converged on the best-adapted (highest payoff) cultural variants. Increased population size increased the rate of convergence on the best-adapted variants, as larger populations had higher initial variation to select from. Crucially, population-level adaptation was moderated by learner type: it was enhanced in populations of maintainers, and impeded in populations of copiers and (more strongly) innovators. The benefit of a maintenance strategy on population-level adaptation was most strongly seen with low-to-moderate payoff bias in smaller populations (see Fig. 3b).

The first set of simulations showed a stronger effect of learner type in small unstructured populations than in large unstructured populations. However, large unstructured populations are not realistic: people socialise in smaller subgroups within larger populations. To address this concern, and to test the robustness of our results, the second set of simulations examined the influence of the different parameter combinations on variant diversity and adaptation in structured populations with neighbourhood sizes of approximately 6, 16, 25 and 33 agents. Population size was held constant at 200 agents. We ran 10,000 simulations for each parameter combination.

Results from the population structure simulations largely mirror the results from the population size simulations. Agents converged on a reduced set of cultural variants over time, and convergence was faster and stronger as payoff bias increased. Populations with larger neighbourhoods showed the strongest convergence (consistent with $\mathrm{Barr}^{55}$ ). Population-level convergence was again moderated by learner type. Again, populations of innovators converged the least; however, in contrast to the unstructured populations, in the structured populations maintainers converged to a similar or higher level than copiers (see Fig. 4a).

Variant adaptation required payoff-biased learning. As payoff bias increased, populations increasingly converged on the best-adapted cultural variants. Increased neighbourhood size increased the rate of convergence on the best-adapted variants, as larger neighbourhoods granted access to more variation to select from. Crucially, population-level adaptation was moderated by learner type: it was enhanced in populations of maintainers, 

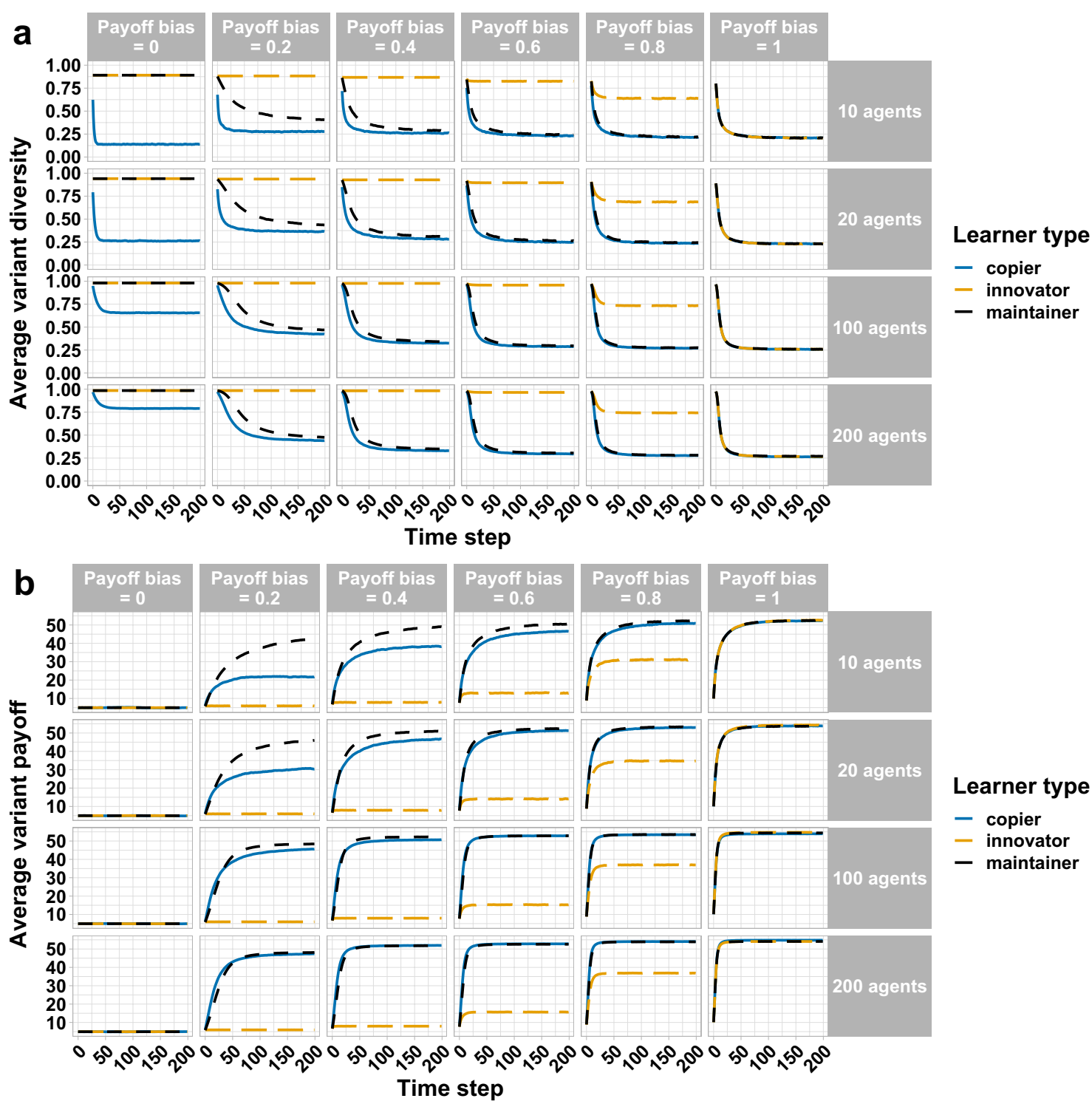

Figure 3. Change in cultural diversity (a) and average variant payoff (adaptation) (b) over 200 time steps as a function of the different parameter combinations (payoff bias, learner type and population size). Populations were unstructured. Variant diversity was measured by the Gini-Simpson index of diversity (maximum for 10-, $20-, 100$ - and 200-agent populations $=0.9,0.95,0.99$ and 0.995 respectively). Each line is the average of 10,000 simulations.

and impeded in populations of copiers and (more strongly) innovators. The benefit of a maintenance strategy on population-level adaptation was most strongly seen with low-to-moderate payoff bias in populations with smaller neighbourhoods (see Fig. 4b).

\section{Discussion}

During social interaction people encounter the cultural variants produced by others, and can compare these to their own cultural variants. A similar process occurs during asocial learning, where people can compare innovated variants to those they already possess. Selection occurs when people adopt one variant and discard the other. This is the premise of the simulations reported in the present paper, where we examined the populationlevel outcomes of this selection process. In line with previous research, we demonstrated that population-level adaptation required agents to be critical, relying on some level of payoff-biased learning ${ }^{34-37}$. Population-level adaptation also benefited from greater access to cultural variation via increased population size or neighbourhood size $^{17,60,61}$. Greater access to cultural variation increased the probability of agents encountering better-adapted variants, and payoff-biased learning increased the probability of these variants being copied and thereby propagating within the population (see also Schlag ${ }^{52,62}$ ). When learners were uncritical and did not base their variant choices on payoffs, copying accelerated convergence by eliminating cultural variation. This involved occasionally overwriting better-adapted variants with worse ones. Uncritical innovation also had this problem, and additionally led 

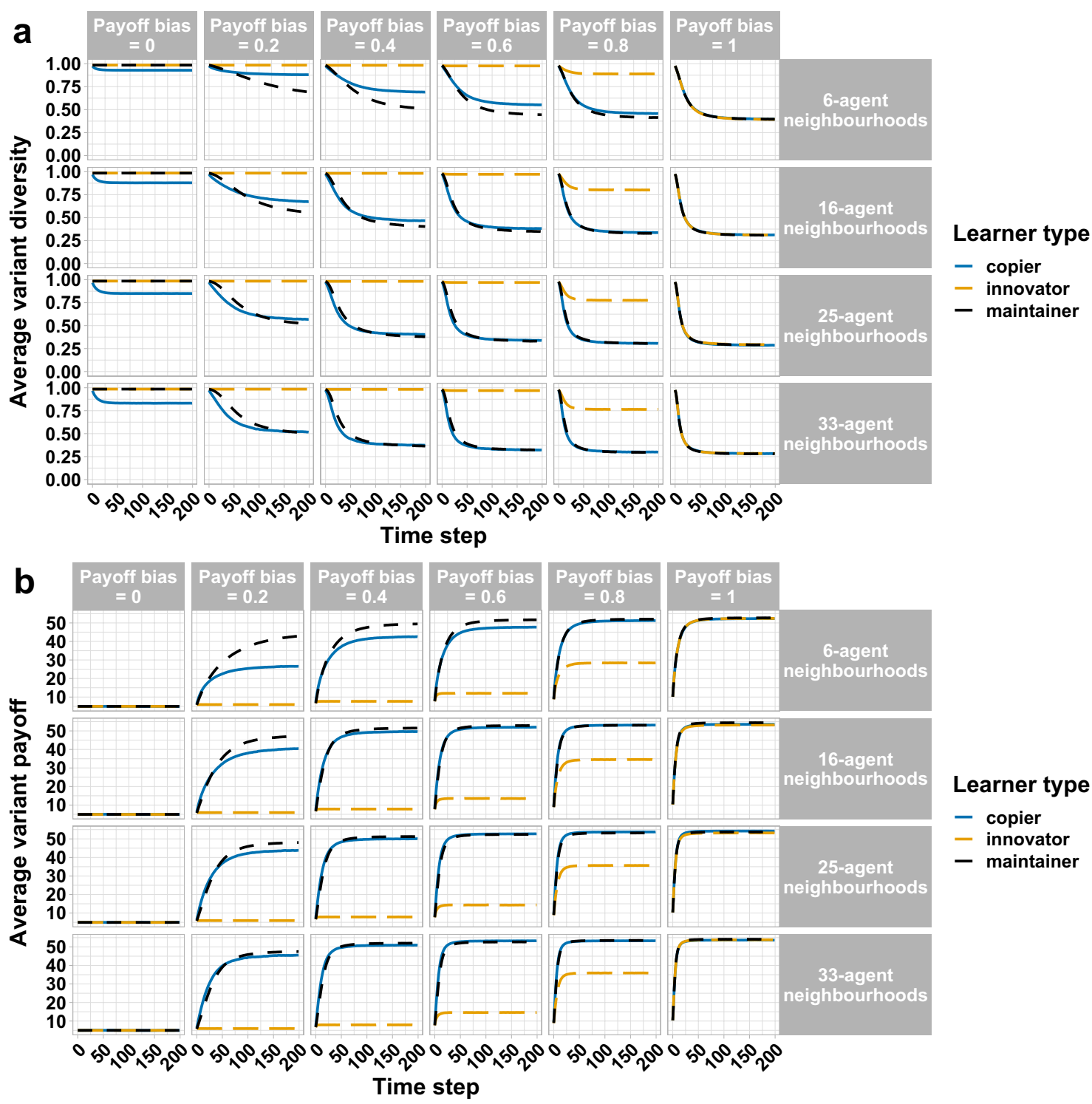

Figure 4. Change in cultural diversity (a) and average variant payoff (adaptation) (b) over 200 time steps as a function of the different parameter combinations (payoff bias, learner type and neighbourhood size). The number of agents in each neighbourhood is approximate (see "Method" section). Each population had 200 agents. Variant diversity was measured by the Gini-Simpson index of diversity (maximum for 200-agent populations $=0.995$ ). Each line is the average of 10,000 simulations.

to the introduction (or reintroduction) of low-payoff variants. Thus copying and innovation as uncritical default strategies disrupted or even reversed the adaptive filtering process. By contrast, uncritical maintenance paused adaptive filtering without disrupting it; cultural variation was retained, slowing convergence, but increasing the retention of better-adapted cultural variants. This improved the environment from which payoff-biased learning could pick out the best-adapted cultural variants, leading to stronger population-level adaptation.

When payoff bias was strongest variant selection was unaffected by the uncritical default behaviours (copying, innovating and maintaining). Weaker payoff bias allowed the default behaviours to influence variant choice. This is why the biggest benefit of maintenance over copying and innovation (and the biggest benefit of copying over innovation) occurred when payoff bias was weak-to-moderate (which may also be more realistic than a stronger level of payoff bias ${ }^{50}$ ). The benefit of maintenance over copying and innovation was most evident in small populations and large populations with small neighbourhood sizes. The loss of adaptive variants due to uncritical copying or innovation was especially detrimental in small populations due to the lower probability of other agents having a "replacement" copy of the discarded variant. Variant replacement was less problematic in larger populations because multiple copies were more likely to co-exist. However, in large populations organized into small neighbourhoods, agents had less access to variants and therefore also ran into the variant replacement problem. This is why population-level adaptation was stronger for maintainers than for copiers or innovators. It is worth noting that the effects of population and neighbourhood size are dependent on the number of possible variants; 
when the number of possible variants is increased the benefit of maintenance extends to the larger population and neighbourhood sizes (see Supplementary Information S2). So, while the population and neighbourhood sizes we simulated can be considered "large" or "small" relative to the number of possible variants (i.e., 100), the absolute sizes cannot be interpreted straightforwardly.

An unexpected finding was that populations of maintainers converged as much, or more than, populations of copiers when population structure was introduced. By contrast, in unstructured populations copiers converged more than maintainers. This difference in convergence can be explained by a combination of uncritical copying and neighbourhood structure restricting the spread of high-payoff variants. In structured populations, uncritical copying could create "pockets" of low-payoff variants. For agents within these pockets, the variants they had access to via maintenance and copying often had lower payoffs than the variants they had access to through innovation. This increased the innovation rate when agents were critical, reintroducing variation into the population and impeding convergence. Unstructured populations of copiers did not experience this to the same degree, as the spread of high-payoff variants from elsewhere in the population was unrestricted and lowpayoff pockets were less likely to form.

Although maintenance of prior behaviour is common in studies of social learning, researchers have struggled to explain why this is the case $\mathrm{e}^{27}$. For instance, some researchers have suggested that people may prefer their own position because they have more complete access to the reasoning behind it $^{26,63}$; however, people overweight positions falsely presented as their own, for which they have no access to the reasoning ${ }^{64}$. Other explanations, such as anchoring effects and culture-specific individualistic values, have been similarly unsupported ${ }^{27}$. Our results suggest another line of explanation: maintenance could be adaptive. More specifically, our simulations suggest that maintenance is safer than copying or innovation when not basing variant choices on critical evaluation. This could support an evolutionary explanation for the ubiquity of maintenance (e.g., where natural selection favours defaulting to maintenance when evaluation is costly), but could also indicate other, more proximate explanations; for instance, if maintenance is adaptive then asocial learning may lead people to rely on it. Future research could explore this line of explanation.

A potential limitation of the reported simulations is that we did not consider any costs that may be incurred by learners (e.g., researchers typically consider asocial learning to be costlier than social learning). We address two types of cost in the Supplementary Information. First, we considered how Darwinian cultural evolution may be affected by an evaluation cost (Supplementary Information S3), which is applied whenever a learner adopts a variant through payoff-biased learning. While payoff-biased learning was again needed for cultural adaptation, when evaluation was costly any extra benefit of more-than-minimal payoff-biased learning was overwhelmed by the cost incurred. As a result, the optimal level of payoff bias in the simulations was low-the same level at which maintainers had the largest advantage over copiers and innovators across all simulations. Second, we considered how Darwinian cultural evolution may be affected by a learning cost (Supplementary Information S4), which is applied whenever a learner adopts a variant through copying or innovation (i.e., where the variant is new so there are costs to learning it, such as time and energy, and the learner has reduced proficiency). Stronger learning costs increased the advantage of maintainers over copiers and innovators, and increased the amount of maintenance occurring in all populations via payoff-biased learning. These results demonstrate that incorporating two types of cost into the simulations boosts the advantage of maintenance over copying and innovation as a default learning strategy.

\section{Conclusion}

Maintenance of prior behaviour, as opposed to copying or innovation, is common during social learning, but has been overlooked in the social learning literature ${ }^{24}$ and researchers have struggled to explain its dominance ${ }^{27}$. The agent-based simulations reported here demonstrate that, when learners do not rely exclusively on critical, payoff-biased learning, maintenance as a "default" uncritical strategy can enhance population-level adaptation compared to copying and innovation. Copying and innovation as uncritical default strategies disrupted the adaptive filtering process underlying Darwinian cultural evolution, by overwriting adaptive variants and introducing maladaptive variants. By contrast, maintenance paused adaptive filtering without disrupting it, retaining filtered variation until it could be further filtered by payoff-biased learning. This led to stronger population-level adaptation, especially when payoff bias was weak-to-moderate and agents were in small populations or large populations organised into small neighbourhoods. These results demonstrate the effect that default uncritical strategies may have on Darwinian cultural evolution. In addition, the benefit of maintenance as a default strategy may explain its dominance in human social learning.

\section{Data availability}

The simulation code and data generated by it are available on the Open Science Framework: https://osf.io/ 6hmg5/?view_only=7dc34448fb774969b99440ba9724ab56.

Received: 24 May 2021; Accepted: 21 September 2021

Published online: 06 October 2021

\section{References}

1. Boyd, R., Richerson, P. J. \& Henrich, J. The cultural niche: Why social learning is essential for human adaptation. Proc. Natl. Acad. Sci. 108, 10918-10925 (2011).

2. Cavalli-Sforza, L. L., Feldman, M. W., Chen, K. H. \& Dornbusch, S. M. Theory and observation in cultural transmission. Science 218, 19-27 (1982).

3. Csibra, G. \& Gergely, G. Natural pedagogy as evolutionary adaptation. Philos. Trans. R. Soc. B Biol. Sci. 366, 1149-1157 (2011).

4. Boyd, R. \& Richerson, P. J. Culture and the Evolutionary Process (University of Chicago Press, 1985). 
5. Cavalli-Sforza, L. L. \& Feldman, M. W. Cultural Transmission and Evolution: A Quantitative Approach (Princeton University Press, 1989).

6. Whiten, A., Hinde, R. A., Laland, K. N. \& Stringer, C. B. Culture evolves. Philos. Trans. R. Soc. B Biol. Sci. 366, 938-948 (2011).

7. Mesoudi, A., Whiten, A. \& Laland, K. N. Perspective: Is human cultural evolution Darwinian? Evidence reviewed from the perspective of The Origin of Species. Evolution 58, 1-11 (2004).

8. Basalla, G. The Evolution of Technology (Cambridge University Press, 1988).

9. Eriksson, K. \& Coultas, J. C. Corpses, maggots, poodles and rats: Emotional selection operating in three phases of cultural transmission of urban legends. J. Cogn. Cult. 14, 1-26 (2014).

10. Nia, H. T. et al. The evolution of air resonance power efficiency in the violin and its ancestors. Proc. R. Soc. Math. Phys. Eng. Sci. 471, 20140905 (2015).

11. Vincenti, W. G. Real-world variation-selection in the evolution of technological form: Historical examples. In Technological Innovation as an Evolutionary Process (ed. Ziman, J.) 174-189 (Cambridge University Press, 2000).

12. Morgan, T. J. H. et al. Experimental evidence for the co-evolution of hominin tool-making teaching and language. Nat. Commun. 6, $6029(2015)$

13. Mesoudi, A. \& O’Brien, M. J. The cultural transmission of Great Basin projectile-point technology I: An experimental simulation. Am. Antiq. 73, 3-28 (2008).

14. Derex, M., Beugin, M.-P., Godelle, B. \& Raymond, M. Experimental evidence for the influence of group size on cultural complexity. Nature 503, 389-391 (2013).

15. Mesoudi, A. An experimental simulation of the "copy-successful-individuals" cultural learning strategy: Adaptive landscapes, producer-scrounger dynamics, and informational access costs. Evol. Hum. Behav. 29, 350-363 (2008).

16. Caldwell, C. A. \& Millen, A. E. Social learning mechanisms and cumulative cultural evolution: Is imitation necessary?. Psychol. Sci. 20, 1478-1483 (2009).

17. Fay, N., De Kleine, N., Walker, B. \& Caldwell, C. A. Increasing population size can inhibit cumulative cultural evolution. Proc. Natl. Acad. Sci. 116, 6726-6731 (2019).

18. Fay, N., Garrod, S. \& Roberts, L. The fitness and functionality of culturally evolved communication systems. Philos. Trans. R. Soc. Lond. B Biol. Sci. 363, 3553-3561 (2008).

19. Kirby, S., Cornish, H. \& Smith, K. Cumulative cultural evolution in the laboratory: An experimental approach to the origins of structure in human language. Proc. Natl. Acad. Sci. 105, 10681-10686 (2008).

20. Fay, N. \& Ellison, T. M. The cultural evolution of human communication systems in different sized populations: Usability trumps learnability. PLoS ONE 8, e71781 (2013).

21. Lyons, D. E., Young, A. G. \& Keil, F. C. The hidden structure of overimitation. Proc. Natl. Acad. Sci. 104, 19751-19756 (2007).

22. Bonaccio, S. \& Dalal, R. S. Advice taking and decision-making: An integrative literature review, and implications for the organizational sciences. Organ. Behav. Hum. Decis. Process. 101, 127-151 (2006).

23. Eriksson, K. \& Strimling, P. Biases for acquiring information individually rather than socially. J. Evol. Psychol. 7, 309-329 (2009).

24. Hodges, B. H. Rethinking conformity and imitation: Divergence, convergence, and social understanding. Front. Psychol. 5, 726 (2014).

25. Mesoudi, A., Chang, L., Murray, K. \& Lu, H. J. Higher frequency of social learning in China than in the West shows cultural variation in the dynamics of cultural evolution. Proc. R. Soc. B Biol. Sci. 282, 20142209 (2015).

26. Yaniv, I. \& Kleinberger, E. Advice taking in decision making: Egocentric discounting and reputation formation. Organ. Behav. Hum. Decis. Process. 83, 260-281 (2000).

27. Morin, O., Jacquet, P. O., Vaesen, K. \& Acerbi, A. Social information use and social information waste. Philos. Trans. R. Soc. B 376, $20200052(2021)$.

28. Harrison, R. A. \& Whiten, A. Chimpanzees (Pan troglodytes) display limited behavioural flexibility when faced with a changing foraging task requiring tool use. PeerJ 6, e4366 (2018).

29. Gruber, T., Muller, M. N., Reynolds, V., Wrangham, R. \& Zuberbühler, K. Community-specific evaluation of tool affordances in wild chimpanzees. Sci. Rep. 1, 128 (2011).

30. Davis, S. J., Vale, G. L., Schapiro, S. J., Lambeth, S. P. \& Whiten, A. Foundations of cumulative culture in apes: Improved foraging efficiency through relinquishing and combining witnessed behaviours in chimpanzees (Pan troglodytes). Sci. Rep. 6, 35953 (2016).

31. Nakahashi, W., Wakano, J. Y. \& Henrich, J. Adaptive social learning strategies in temporally and spatially varying environments. Hum. Nat. 23, 386-418 (2012).

32. McElreath, R. et al. Beyond existence and aiming outside the laboratory: Estimating frequency-dependent and pay-off-biased social learning strategies. Philos. Trans. R. Soc. B Biol. Sci. 363, 3515-3528 (2008).

33. Kendal, J., Giraldeau, L.-A. \& Laland, K. The evolution of social learning rules: Payoff-biased and frequency-dependent biased transmission. J. Theor. Biol. 260, 210-219 (2009).

34. Ehn, M. \& Laland, K. Adaptive strategies for cumulative cultural learning. J. Theor. Biol. 301, 103-111 (2012).

35. Enquist, M., Eriksson, K. \& Ghirlanda, S. Critical social learning: A solution to Rogers's paradox of nonadaptive culture. Am. Anthropol. 109, 727-734 (2007).

36. Kameda, T. \& Nakanishi, D. Does social/cultural learning increase human adaptability?: Rogers's question revisited. Evol. Hum. Behav. 24, 242-260 (2003).

37. Rendell, L., Fogarty, L. \& Laland, K. N. Rogers' paradox recast and resolved: Population structure and the evolution of social learning strategies. Evolution 64, 534-548 (2010).

38. Rogers, A. R. Does biology constrain culture?. Am. Anthropol. 90, 819-831 (1988).

39. Rogers, S. L. \& Fay, N. Stick or switch: A selection heuristic predicts when people take the perspective of others or communicate egocentrically. PLOS ONE 11, e0159570 (2016).

40. McElreath, R. et al. Applying evolutionary models to the laboratory study of social learning. Evol. Hum. Behav. 26, 483-508 (2005).

41. Kendal, R. L. et al. Social learning strategies: Bridge-building between fields. Trends Cogn. Sci. 22, 651-665 (2018).

42. Laland, K. N. Social learning strategies. Anim. Learn. Behav. 32, 4-14 (2004).

43. Chudek, M., Heller, S., Birch, S. \& Henrich, J. Prestige-biased cultural learning: Bystander's differential attention to potential models influences children's learning. Evol. Hum. Behav. 33, 46-56 (2012).

44. Atkisson, C., O’Brien, M. J. \& Mesoudi, A. Adult learners in a novel environment use prestige-biased social learning. Evol. Psychol. 10, $147470491201000320(2012)$.

45. Haun, D. B. M., Rekers, Y. \& Tomasello, M. Majority-biased transmission in chimpanzees and human children, but not orangutans. Curr. Biol. 22, 727-731 (2012).

46. Lewandowsky, S., Cook, J., Fay, N. \& Gignac, G. E. Science by social media: Attitudes towards climate change are mediated by perceived social consensus. Mem. Cognit. 47, 1445-1456 (2019).

47. van den Berg, P., Molleman, L. \& Weissing, F. J. Focus on the success of others leads to selfish behavior. Proc. Natl. Acad. Sci. 112, 2912-2917 (2015).

48. Chudek, M., Baron, A. S. \& Birch, S. Unselective overimitators: The evolutionary implications of children's indiscriminate copying of successful and prestigious models. Child Dev. 87, 782-794 (2016).

49. Tamariz, M. Replication and emergence in cultural transmission. Phys. Life Rev. 30, 47-71 (2019). 
50. Tamariz, M., Ellison, T. M., Barr, D. J. \& Fay, N. Cultural selection drives the evolution of human communication systems. Proc. R. Soc. Lond. B Biol. Sci. 281, 20140488 (2014).

51. Rendell, L. et al. Why copy others? Insights from the social learning strategies tournament. Science 328, 208-213 (2010).

52. Schlag, K. H. Why imitate, and if so, how?: A boundedly rational approach to multi-armed bandits. J. Econ. Theory 78, 130-156 (1998).

53. Fay, N., Garrod, S., Roberts, L. \& Swoboda, N. The interactive evolution of human communication systems. Cogn. Sci. 34, 351-386 (2010).

54. Segovia-Martín, J., Walker, B., Fay, N. \& Tamariz, M. Network connectivity dynamics, cognitive biases, and the evolution of cultural diversity in round-robin interactive micro-societies. Cogn. Sci. 44, e12852 (2020).

55. Barr, D. J. Establishing conventional communication systems: Is common knowledge necessary?. Cogn. Sci. 28, 937-962 (2004).

56. Simpson, E. H. Measurement of diversity. Nature 163, 688 (1949).

57. Acerbi, A., Jacquet, P. O. \& Tennie, C. Behavioral constraints and the evolution of faithful social learning. Curr. Zool. 58, 307 (2012).

58. Enquist, M., Strimling, P., Eriksson, K., Laland, K. \& Sjostrand, J. One cultural parent makes no culture. Anim. Behav. 79, 1353-1362 (2010).

59. Kandler, A. \& Laland, K. N. An investigation of the relationship between innovation and cultural diversity. Theor. Popul. Biol. 76, 59-67 (2009).

60. Henrich, J. Demography and cultural evolution: How adaptive cultural processes can produce maladaptive losses: The Tasmanian case. Am. Antiq. 69, 197-214 (2004).

61. Kobayashi, Y. \& Wakano, J. Y. Evolution of social versus individual learning in an infinite island model. Evolution 66, 1624-1635 (2012).

62. Schlag, K. H. Which one should I imitate?. J. Math. Econ. 31, 493-522 (1999).

63. Yaniv, I. Receiving other people's advice: Influence and benefit. Organ. Behav. Hum. Decis. Process. 93, 1-13 (2004).

64. Harvey, N. \& Harries, C. Effects of judges' forecasting on their later combination of forecasts for the same outcomes. Int. J. Forecast. 20, 391-409 (2004).

\section{Acknowledgements}

N.F. and B.W. were funded by the Defence Science and Technology (DST) Group under a Collaborative Agreement 8382 .

\section{Author contributions}

B.W. and N.F. conceived the study. B.W. programmed the simulations and wrote the original draft. N.F. contributed to the writing. J.S.M. and M.T. provided feedback on the simulations. All authors reviewed the manuscript.

\section{Competing interests}

The authors declare no competing interests.

\section{Additional information}

Supplementary Information The online version contains supplementary material available at https://doi.org/ 10.1038/s41598-021-99340-7.

Correspondence and requests for materials should be addressed to N.F.

Reprints and permissions information is available at www.nature.com/reprints.

Publisher's note Springer Nature remains neutral with regard to jurisdictional claims in published maps and institutional affiliations.

(c) (i) Open Access This article is licensed under a Creative Commons Attribution 4.0 International cc) License, which permits use, sharing, adaptation, distribution and reproduction in any medium or format, as long as you give appropriate credit to the original author(s) and the source, provide a link to the Creative Commons licence, and indicate if changes were made. The images or other third party material in this article are included in the article's Creative Commons licence, unless indicated otherwise in a credit line to the material. If material is not included in the article's Creative Commons licence and your intended use is not permitted by statutory regulation or exceeds the permitted use, you will need to obtain permission directly from the copyright holder. To view a copy of this licence, visit http://creativecommons.org/licenses/by/4.0/.

(C) The Author(s) 2021 